\title{
Check-list das Angiospermas do Chaco de Mato Grosso do Sul
}

\author{
Ângela Lúcia Bagnatori Sartori, Vali Joana Pott, Arnildo Pott \& Fábia Silva de Carvalho
}

Universidade Federal de Mato Grosso do Sul, Centro de Ciências Biológicas e da Saúde, Cidade Universitária, CEP 79070-900, Caixa Postal 549; Campo Grande, MS, Brasil. albsartori@gmail.com

Recebido em 27.XI.2014

Aceito em 30.V.2016

DOI 10.21826/2446-8231201873s22

RESUMO - Este check-list foi pautado em dados oriundos de estudos sistematizados realizados no Chaco brasileiro, nos últimos seis anos. Foram registrados 388 táxons, distribuídos em 67 famílias e 254 gêneros. Leguminosae (95 spp.), Poaceae (45 spp.), Asteraceae (27 spp.), Malvaceae (25 spp.), Euphorbiaceae (15 spp.), Apocynaceae (13 spp.) e Rubiaceae (nove spp.) detém 58\% da riqueza. Nossos dados adicionam 68 táxons à lista da flora brasileira, com destaque para Bauhinia hagenbeckii Harms, recoletada após longos 90 anos e apenas conhecida da coleta do tipo. Muellera nudiflora (Burkart) M.J. Silva \& A.M.G. Azevedo, sem informação precisa dos locais de ocorrência quando estabelecida, também foi confirmada para o Chaco brasileiro. Destacamos que coletas efetuadas ao oeste de Mato Grosso do Sul, muitas vezes, correspondem às formações chaquenhas, no entanto, a designação destes locais como pertencentes ao Cerrado e Pantanal inviabiliza o entendimento da distribuição geográfica de muitas espécies que devem formar uma unidade com as demais áreas de Chaco da América do Sul.

Palavras-chave: América do Sul, flora, florestas secas, Pantanal

ABSTRACT - Checklist of Angiosperm from the Chaco of Mato Grosso do Sul. The checklist was obtained from systematic studies undertaken in the Brazilian Chaco over the last six years. We recorded 388 taxa, distributed in 67 families and 254 genera. Leguminosae (95 spp.), Poaceae (45 spp.), Asteraceae (27 spp.), Malvaceae (25 spp.), Euphorbiaceae (15 spp.), Apocynaceae (13 spp.) and Rubiaceae (nine spp.) amounted to $58 \%$ of the richness. Our data added 68 taxa to the list of Flora of Brazil, highlighting Bauhinia hagenbeckii collected again after 90 years and only known from the type collection. Muellera nudiflora without precise information on occurrence when established also was confirmed for the Brazilian Chaco. The collections carried out of the southwestern of the Mato Grosso do Sul often correspond to chaquenian formations, however, the designation of these sites as belonging to the Cerrado and Pantanal hinders understanding of the geographic distribution of many species that must form a unit with the other areas of Chaco in South America.

Keywords: dry forests, flora, Pantanal, South America

\section{INTRODUÇÃO}

As formações chaquenhas exclusivas da América do Sul extendem-se nas planícies ao norte da Argentina, oeste do Paraguai, sudeste da Bolívia e ao oeste do Brasil, totalizando cerca de $1000.000 \mathrm{~km}^{2}$ (Zak \& Cabido 2002). Em território brasileiro tais formações ocupam aproximadamente 70.000 $\mathrm{km}^{2}$ e penetram como uma estreita faixa paralela ao rio Paraguai, no Mato Grosso do Sul (Hueck 1972, Prado 1993).

No Brasil, as formações chaquenhas à semelhança das registradas para a Argentina e Paraguai ocorrem em maiores extensões em Porto Murtinho, no Mato Grosso do Sul (Prado et al. 1992). Essas formações recebem influência das Florestas Estacionais e do Cerrado, o que pode dificultar, às vezes, o pronto reconhecimento das mesmas (Sartori 2012). Em termos florísticos poucos estudos foram realizados no Chaco brasileiro (Nunes 2006, Noguchi et al. 2009, Alves 2008, Alves \& Sartori 2009, Salomão 2009, Seleme 2009), porém fundamentais para o registro de espécies de ocorrência restrita, novas ocorrências e de informações precisas sobre a biologia das mesmas. Somado a isso as áreas de Chaco no Brasil em acelerado processo de fragmentação (Silva et al. 2008) têm recebido nas últimas décadas importante pressão antrópica, o que coloca em risco a biodiversidade da região.

A compilação de dados florísticos oriunda de estudos sistematizados realizados nos últimos anos no Chaco brasileiro, em Porto Murtinho, é oportuna para disponibilizar à comunidade científica o acúmulo de conhecimento gerado (Nunes 2006, Noguchi et al. 2009, Alves 2008, Alves \& Sartori 2009, Salomão 2009, Seleme 2009, Padilha 2011, Carvalho 2011), pois até o momento inexiste uma estimativa da riqueza de espécies de Angiospermas para as formações chaquenhas brasileiras.

\section{MATERIAL E MÉTODOS}

O checklist das famílias foram obtidos a partir de consulta ao Herbário CGMS e complementados com consultas aos bancos de dados de herbários disponíveis em rede. Ressalta-se que na listagem foram incluídos os vouchers oriundos do Chaco localizado no município de Porto Murtinho, pois segundo Prado et al. (1992) se trata do mais próximo das formações de Chaco do Paraguai e Argentina. Dentre os subtipos de Chaco descritos pelo IBGE (1992) com base na fitofisionomia e determinados elementos florísticos predominantes têm-se: Savana Estépica Florestada ou Chaco Florestado, Savana Estépica 
Arborizada ou Chaco Arborizado, Savana Estépica Parque ou Carandazal e Savana Estépica Gramíneo-Lenhosa ou Chaco com campo nativo. As informações foram oriundas da Savana Estépica Florestada (Chaco Florestado) e Savana Estépica Arborizada (Chaco Arborizado), sensu IBGE (1992).

A apresentação das famílias seguiu a ordem alfabética e foi baseada na proposta de classificação das Angiospermas, conforme o APG III (2009). A checagem e atualização dos nomes botânicos foram baseadas na Lista de Espécies da Flora do Brasil (2012) e CRIA (2012).

\section{RESULTADOS E DISCUSSÃO}

Foram registrados 388 táxons distribuídos em 67 famílias e 252 gêneros. As famílias mais representativas foram: Leguminosae (95 spp.), Poaceae (45 spp.), Asteraceae (27 spp.), Malvaceae (24 spp.), Euphorbiaceae (14 spp.), Apocynaceae (13 spp.) e Rubiaceae (nove spp.), o que representa $58 \%$ da riqueza específica concentrada em sete famílias botânicas; 38 famílias foram representadas por uma a duas espécies e as demais por menos de 10 espécies. Leguminosae, Poaceae, Euphorbiaceae também se destacaram em riqueza em diferentes estudos para o Chaco brasileiro (Noguchi et al. 2009, Nunes 2006, Salomão 2010, Seleme 2010, Carvalho 2011) e para o argentino (Marino \& Pensiero 2003, Roic et al. 1999, Prado et al. 1992).

Com base no checklist verificamos a ocorrência de 68 táxons com novas ocorrências para o Brasil se considerarmos como referencial a Lista de Espécies da Flora do Brasil 2012 (in http://floradobrasil.jbrj. gov.br/2012). No entanto, algumas espécies não foram registradas na lista, porém constam na literatura como elementos do Chaco (Lorenzi 1998, Lorenzi 2009, Pott \& Pott 1994, Pott \& Pott 1999), como Prosopis nigra (Griseb.) Hieron., Prosopis rubriflora Hassl. Prosopis ruscifolia Griseb, Piptadenia viridiflora (Kunth) Benth., Mimosa glutinosa Malme, Guibourtia hymenifolia (Moric.) J. Léonard, Libidibia paraguariensis (D. Parodi) G.P. Lewis, Ceiba samauma (Mart.) K. Schum., Tabebuia nodosa (Gris) Gris., Aspidosperma quebracho-blanco Schltdl., Aspidosperma pyrifolium Mart., Schinopsis balansae Engl., Bulnesia sarmentoi Lorentz ex Griseb., Phyllanthus chacoensis Morong, Diplokeleba floribunda N.E. Br., Ruprechtia exploratricis Sandwith, dentre outras.

Espécies registradas para o Chaco argentino e paraguaio (Peña-Chocarro et al. 2006) também ocorrem no brasileiro a exemplo de Prosopis alba Griseb., Prosopis nigra, Prosopis ruscifolia, Parkinsonia praecox (Ruiz \& Pav.) J. Hawkins, Tabebuia nodosa, Schinopsis brasiliensis Engl., Aspidosperma quebrachoblanco, Ziziphus mistol Griseb., Phyllanthus chacoensis, Grabowskia duplicata Arn., Diplokeleba floribunda,
Bulnesia sarmentoi, Albizia inundata (Mart.) Barneby \& J.W.Grimes, Libidibia paraguariensis, Parapiptadenia rigida (Benth.) Brenan, além de espécies de Capparaceae. Como parte do grupo de espécies de formações chaquenhas somam-se ainda no Chaco brasileiro Echinopsis rhodotricha K. Schum., Tillandsia didisticha (E. Morren) Baker, Tillandsia duratii Vis., Castela coccinea Griseb., Aspidosperma triternatum Rojas Acosta e Bauhinia hagenbeckii Harms. Bulnesia sarmentoi, até o presente sem voucher disponível na rede speciesLink corresponde a uma espécie com poucos indivíduos nas formações chaquenhas de MS, possivelmente devido à exploração da madeira para fins comerciais. Bauhinia hagenbeckii não havia sido mais coletada desde o seu estabelecimento em 1905. Vaz \& Tozzi (2005) ao efetuar a revisão do gênero citou a espécie para o Chaco sem dados precisos do local de ocorrência. Muellera nudiflora (Burkart) M.J. Silva \& A.M.G. Azevedo com distribuição restrita no Brasil (Silva \& Tozzi 2015) foi citada apenas para o Pantanal. Ambas espécies ocorrem no Chaco brasileiro (Carvalho 2011) e populações destas foram localizadas nos últimos anos no MS.

Informações mais precisas sobre o habitat de muitas espécies que integram o checklist poderão ser referenciadas como também presentes no Chaco brasileiro, a exemplo de Gymnocoronis spilanthoides DC. citada como de domínio desconhecido (Almeida 2012). A citação de ocorrência para o Cerrado de MS (Lista de Espécies da Flora do Brasil 2012 in http://floradobrasil.jbrj.gov.br/2012) de Schinopsis balansae, S. brasiliensis, Annona nutans (R.E. Fr.) R.E. Fr., Aspidosperma quebracho-blanco, Rauvolfia ligustrina Willd. ex Roem. \& Schult., Pterocaulon purpurascens Malme, Tillandsia didisticha, Geoffrea spinosa Jacq. e para o Pantanal (Lista de Espécies da Flora do Brasil 2012 in http://floradobrasil.jbrj.gov.br/2012) de Tabebuia nodosa, Anisocapparis speciosa Griseb. Cornejo \& Iltis, Cynophalla retusa (Griseb.) Cornejo \& Iltis, Libidibia paraguariensis, Muellera nudiflora, Parkinsonia aculeata L., Pavonia laetevirens R.E. Fr., dentre outras, é pouco informativa, o que não reflete as observações de campo. Salientamos a necessidade de revisão dos termos que se referem aos domínios brasileiros com a inclusão do Chaco na região oeste do MS, pois agrega elementos florísticos que conferem unidade às formações chaquenhas da América do Sul.

Ações que visem à preservação do Chaco brasileiro, considerado uma área de elevada diversidade biológica (MMA 2002), se fazem necessárias, pois os remanescentes em território sul-mato-grossense estão desaparecendo rapidamente nos últimos anos. A criação de uma Unidade de Conservação no município de Porto Murtinho deve ser encorajada com vistas a proteger maior área possível de Chaco, único no Brasil. 
Quadro 1. Check-list das Angiospermas do Chaco de Mato Grosso do Sul (*táxons não citados no site da Flora do Brasil para o MS).

\begin{tabular}{|c|c|}
\hline Família/Espécie & Voucher \\
\hline \multicolumn{2}{|l|}{ Acanthaceae } \\
\hline Justicia laevilinguis (Nees) Lindau & Salomão, A.K.D. 451 (CGMS) \\
\hline Ruellia hygrophila Mart. & Salomão, A.K.D. 304 (CGMS) \\
\hline \multicolumn{2}{|l|}{ Alismataceae } \\
\hline Hydrocleys nymphoides (Willd.) Buchenau & Lescano, L.E.A.M. et al. 248 (CGMS) \\
\hline Sagittaria montevidensis Cham. \& Schltdl. * & Nunes, G.P. et al. 152 (CGMS) \\
\hline \multicolumn{2}{|l|}{ Amaranthaceae } \\
\hline Amaranthus viridis L. & Lescano, L.E.A.M. et al. 286 (CGMS) \\
\hline Gomphrena celosioides Mart. & Alves, F. M. et al. 382 (CGMS) \\
\hline G. celosioides var. aureiflora Stuchl. & Noguchi, D. K. et al. 155 (CGMS) \\
\hline G. elegans Mart. & Alves, F. M. et al. 383 (CGMS) \\
\hline Pfaffia glomerata (Spreng.) Pedersen & Salomão, A.K.D. 448 (CGMS), Alves, F. M. et al. 380 (CGMS) \\
\hline \multicolumn{2}{|l|}{ Amaryllidaceae } \\
\hline Zephyranthes lactea $\mathrm{S}$. Moore & Nunes, G.P. et al. 291 (CGMS) \\
\hline \multicolumn{2}{|l|}{ Anacardiaceae } \\
\hline Myracrodruon urundeuva Allemão & Sartori, A.L.B 1007 (CGMS) \\
\hline Schinopsis balansae Engl. & Seleme, E.P. \& Sartori, A.L.B. 187 (CGMS) \\
\hline S. brasiliensis Engl.* & Pinto, A.S.C. 05 (CGMS), Sartori, A.L.B. et al. 1029 (CGMS) \\
\hline \multicolumn{2}{|l|}{ Annonaceae } \\
\hline Annona nutans (R.E.Fr.) R.E.Fr. & $\begin{array}{l}\text { Seleme, E.P. } 153 \text { (CGMS), Sartori, A.L.B. \& Alves, F.M.1064 } \\
\text { (CGMS) }\end{array}$ \\
\hline \multicolumn{2}{|l|}{ Apiaceae } \\
\hline Eryngium ebracteatum Lam. & Noguchi, D.K. et al. 162 (CGMS), Salomão, A.K.D. 473 (CGMS) \\
\hline \multicolumn{2}{|l|}{ Apocynaceae } \\
\hline Araujia variegata (Griseb.) Fontella \& Gouder & Seleme, E.P. Sartori \& A.L.B. 157 (CGMS), Moro, M.R. 01 (CGMS) \\
\hline A. odorata (Hook. \& Arn.) Fontella \& Goyder & Sartori, A.L.B. 1063 \& Alves, F.M. (CGMS) \\
\hline Asclepias mellodora A.St.Hil. & Sartori, A.L.B. 1080 (CGMS) \\
\hline Aspidosperma pyrifolium Mart. & Maier, J. 02 (CGMS) \\
\hline A. quebracho-blanco Schltdl. & Sartori, A.L.B. et al. 1020a (CGMS) \\
\hline A. triternatum Rojas Acosta & Seleme, E.P. \& Sartori, A.L.B. 181 (CGMS) \\
\hline Funastrum clausum (Jacq.) Schltr. & Lescano, L.E.A.M. et al. 181 (CGMS) \\
\hline Gonolobus rostratus (Vahl) R.Br. ex Shult. & Lescano, L.E.A.M. et al. 59 (CGMS) \\
\hline Mandevilla angustifolia (Malme) Woodson * & Farinaccio, M.A. 896 (CGMS) \\
\hline Oxypetalum balansae Malme & Nunes, G.P. et al. 204 (CGMS) \\
\hline Rauvolfia ligustrina Willd. ex Roem. \& Schult. & $\begin{array}{l}\text { Sartori, A.L.B. (CGMS 23099), Seleme, E.P. \& Sartori, A.L.B. } 108 \\
\text { (CGMS) }\end{array}$ \\
\hline Rhabdadenia madida (Vell.) Miers * & Alves, F. M.52 (CGMS) \\
\hline Schubertia grandiflora Mart. \& Zucc. & Alves, F.M. \& Sartori, A.L.B. 48 (CGMS) \\
\hline \multicolumn{2}{|l|}{ Arecaceae } \\
\hline Copernicia alba Morong ex Morong \& Britton & Seleme, E.P. 398 (CGMS) \\
\hline \multicolumn{2}{|l|}{ Aristolochiaceae } \\
\hline Aristolochia esperanzae Kuntze & Sartori, A.L.B. et al. 1060 (CGMS) \\
\hline A. rojasiana (Chodat \& Hassler) F. Gonzalez & Sartori, A.L.B. et al. 1066 (CGMS) \\
\hline \multicolumn{2}{|l|}{ Asteraceae } \\
\hline Barrosoa candolleana (Ho . \& Arn.) R.M. King \& H. Rob. & Noguchi, D.K. et al. 67 (CGMS) \\
\hline Baccharis dracunculifolia DC. & Lescano, L.E.A.M. et al. 20 (CGMS) \\
\hline Calea candolleana Baker * & Nunes, G.P. et al. 52 (CGMS) \\
\hline Centratherum punctatum Cass. & Nunes, G.P. et al. 34 (CGMS) \\
\hline Chaptalia integerrima (Vell.) Burkart * & Lescano, L.E.A.M. et al. 17 (CGMS) \\
\hline Chromolaena lilacina (Hieron) R.M. King \& H. Rob. * & Lescano, L.E.A.M. et al. 213 (CGMS) \\
\hline C. mattogrossensis (Hieron) R. M. King \& H. Rob. * & Nunes, G.P. et al. 84 (CGMS) \\
\hline C. picta (Gardner) R.M.King \& H. Rob. * & D.K. Noguchi et al. 27 (CGMS) \\
\hline Conyza bonariensis (L.) Cronquist & Nunes, G.P. et al. 187 (CGMS) \\
\hline Elephantopus mollis Kunth * & Nunes, G.P. et al. 85 (CGMS) \\
\hline Gymnocoronis spilanthoides DC. & Alves, F. M. et al. 358 (CGMS), Oliveira, L.V.L. 03 (CGMS) \\
\hline Isostigma hoffmannii O.Kuntze * & Nunes, G.P. et al. 117 (CGMS) \\
\hline Lagascea mollis Cav. & D.K. Noguchi et al. 186 (CGMS) \\
\hline Lepidaploa helophila (Mart. ex DC.) H.Rob. * & Noguchi, D.K. et al. 26 (CGMS) \\
\hline
\end{tabular}


Quadro 1. Cont.

\begin{tabular}{|c|c|}
\hline Família/Espécie & Voucher \\
\hline L. muricata (DC.) H.Rob. * & Nunes, G.P. et al. 136 (CGMS) \\
\hline L. reflexa (Gardner) H.Rob. * & D.K. Noguchi et al. 35 (CGMS) \\
\hline Lessingianthus rubricaulis (Humb. \& Bonpl.) H.Rob. & $\begin{array}{l}\text { Lescano, L.E.A.M. et al. } 208 \text { (CGMS), Lescano, L.E.A.M et al. } 27 \\
\text { (CGMS) }\end{array}$ \\
\hline Mikania campanulata Gardner * & Lescano, L.E.A.M. et al. 295 (CGMS) \\
\hline Pectis gardneri Baker * & Alves, F. M. et al. 402 (CGMS) \\
\hline Pluchea sagittalis (Lam.) Cabrera * & Lescano, L.E.A.M. et al. 291 (CGMS) \\
\hline Porophyllum ruderale (Jacq.) Cass. & Noguchi, D.K. et al. 07 (CGMS) \\
\hline Pterocaulon alopecuroides (Lam.) DC. & Nunes, G.P. et al. 43 (CGMS) \\
\hline P. lorentzii Malme & Lescano, L.E.A.M. et al. 226 (CGMS) \\
\hline P. purpurascens Malme * & Alves, F.M. et al. 439 (CGMS) \\
\hline Vernonanthura brasiliana (L.) H.Rob. & Nunes, G.P. et al. 39 (CGMS) \\
\hline Vernonanthura petiolaris (DC.) H.Rob. & Lescano, L.E.A.M. et al. 288 (CGMS) \\
\hline Wedelia trichostephia DC. & Salomão, A.K.D. \& Alves, F.M. 369 (CGMS) \\
\hline \multicolumn{2}{|l|}{ Begoniaceae } \\
\hline Begonia cucullata Willd. & Nunes, G.P. et al. 244 (CGMS) \\
\hline \multicolumn{2}{|l|}{ Bignoniaceae } \\
\hline Dolichandra cynanchoides Cham. * & Alves, F.M. et al. 355 (CGMS) \\
\hline Fridericia florida (DC.) L.G.Lohmann & Nunes, G.P. et al. 74 (CGMS) \\
\hline Tabebuia aurea Humb. \& Bonpl. & Lescano, L.E.A.M. et al. 13 (CGMS) \\
\hline T. nodosa (Griseb.) Griseb. & $\begin{array}{l}\text { Lescano, L.E.A.M. et al. } 46 \text { (CGMS), Alves, F.M. et al. } 437 \text { (CGMS), } \\
\text { Sartori, A.L.B. et al. s.n. (CGMS 23101) }\end{array}$ \\
\hline Handroanthus impetiginosus Mattos & Lescano, L.E.A.M. et al. 240 (CGMS) \\
\hline H. ochraceus (Cham.) Mattos & Sartori, A.L.B. et al. s.n. (CGMS 23097) \\
\hline \multicolumn{2}{|l|}{ Bixaceae } \\
\hline Cochlospermum regium (Mart. ex Schrank) Pilg. & Nunes, G.P. et al. 133 (CGMS) \\
\hline \multicolumn{2}{|l|}{ Boraginaceae } \\
\hline Cordia glabrata (Mart.) A.DC. & Nunes, G.P. et al. 86 (CGMS) \\
\hline Heliotropium indicum $\mathrm{L}$. & Lescano, L.E.A.M. et al. 287 (CGMS) \\
\hline H. transalpinum Vell. & Noguchi, D.K. et al. 14 (CGMS) \\
\hline Varronia curassavica Jacq. * & $\begin{array}{l}\text { Seleme, E.P. et al. } 302 \text { (CGMS), Seleme, E.P. \& Sartori, A.L.B. } 196 \\
\text { (CGMS) }\end{array}$ \\
\hline \multicolumn{2}{|l|}{ Bromeliaceae } \\
\hline Bromelia balansae Mez & Salomão, A.K.D. \& Pott, A. 456 (CGMS) \\
\hline B. hieronymii $\mathrm{Mez} *$ & Salomão, A.K.D. \& Pott, A. 439 (CGMS) \\
\hline Tillandsia didisticha (E. Morren) Baker & $\begin{array}{l}\text { Noguchi, D.K. et al. } 231 \text { (CGMS), Salomão, A.K.D. \& Pott, A. } 442 \\
\text { (CGMS) }\end{array}$ \\
\hline T. loliacea Mart. ex Schult. f. & Salomão, A.K.D. \& Pott, A. 402 (CGMS) \\
\hline T. duratii Vis. & Pott, A. 829 (MBM) \\
\hline \multicolumn{2}{|l|}{ Burseraceae } \\
\hline Protium heptaphyllum (Aubl.) Marchand & Noguchi, D.K. et al. 19 (CGMS) \\
\hline \multicolumn{2}{|l|}{ Cactaceae } \\
\hline Cereus stenogonus Rizzini \& A. Mattos & Salomão, A.K.D. \& Pott, A. 474 (CGMS) \\
\hline Cleistocactus baumannii (Lem.) Lem. & Souza, C.S. et al. 60 (CGMS) \\
\hline Echinopsis rhodotricha $\mathrm{K}$. Schum. & Salomão, A.K.D. \& Pott, A. 383 (CGMS) \\
\hline Harrisia balansae (K. Schum.) N.P. Taylor \& Zappi & Arakaki, L.M.M. 17 (CGMS) \\
\hline Opuntia retrorsa F.A.C. Weber * & Salomão, A.K.D. \& Alves, F.M. 311 (CGMS) \\
\hline Pereskia sacharosa Gris. & Noguchi, D.K. et al. 229 (CGMS) \\
\hline Rhipsalis baccifera (J.S. Muell.) Stearn * & Salomão, A.K.D. \& Pott, A. 420 (CGMS) \\
\hline Stetsonia coryne (Salm-Dyck) Britton \& Rose & Farinaccio, M.A. 960 (CGMS) \\
\hline \multicolumn{2}{|l|}{ Cannabaceae } \\
\hline Celtis pubescens Spreng. & Noguchi, D.K. et al. 236 (CGMS) \\
\hline \multicolumn{2}{|l|}{ Capparaceae } \\
\hline Anisocapparis speciosa (Griseb.) Cornejo \& Iltis & Sartori, A.L.B. et al. 1038 (CGMS) \\
\hline Capparicordis tweediana (Eichler) Iltis \& Cornejo & $\begin{array}{l}\text { Seleme, E.P. et al. } 368 \text { (CGMS), Seleme, E.P. \& Sartori, A.L.B. } 234 \\
\text { (CGMS) }\end{array}$ \\
\hline Crateva tapia $\mathrm{L}$. & Seleme, E.P. \& Guanabara 449 (CGMS), Vicente, M.R. 04 (CGMS) \\
\hline Cynophalla retusa (Griseb.) Cornejo \& Iltis & $\begin{array}{l}\text { Padilha, D.R.C. } 26 \text { (CGMS), Seleme, E.P. \& Sartori, A.L.B. } 144 \\
\text { (CGMS) }\end{array}$ \\
\hline
\end{tabular}


Quadro 1. Cont.

\begin{tabular}{|c|c|}
\hline Família/Espécie & Voucher \\
\hline Physostemon guianense (Aubl.) Malme * & Alves, F.M. et al. 392 (CGMS) \\
\hline Tarenaya spinosa (Jacq.) Raf. * & Salomão, A.K.D. \& Pott, A. 459 (CGMS) \\
\hline \multicolumn{2}{|l|}{ Celastraceae } \\
\hline Maytenus ilicifolia Mart. ex Reissek & Seleme, E.P. \& Vacari, A. 412 (CGMS) \\
\hline \multicolumn{2}{|l|}{ Commelinaceae } \\
\hline Commelina schomburgkiana Klotzsch & Salomão, A.K.D. \& Alves, F.M. 297 (CGMS) \\
\hline Murdannia nudiflora (L.) Brenan & Salomão, A.K.D. \& Alves, F.M. 309 (CGMS) \\
\hline Tripogandra glandulosa (Seub.) Rohweder & Salomão, A.K.D. \& Alves, F.M. 358 (CGMS) \\
\hline \multicolumn{2}{|l|}{ Convolvulaceae } \\
\hline Evolvulus filipes Mart. & Salomão, A.K.D. \& Alves, F.M. 324 (CGMS) \\
\hline E. nummularius (L.) L. & Voucher:Noguchi, D.K. et al. 169 (CGMS) \\
\hline E. sericeus $\mathrm{Sw}$. & Lescano, L.E.A.M. et al. 68 (CGMS) \\
\hline Ipomoea carnea Jacq. & $\begin{array}{l}\text { Lescano, L.E.A.M. et al. } 154 \text { (CGMS), Noguchi, D.K. et al. } 189 \\
\text { (CGMS) }\end{array}$ \\
\hline I.chiliantha Hallier f. * & Seleme, E.P. \& Salomão, A.K.D. 395 (CGMS) \\
\hline I. maurandioides Meisn. * & Lescano, L.E.A.M. et al. 132 (CGMS) \\
\hline Merremia aegyptia (L.) Urb. & Nunes, G.P. et al. 53 (CGMS) \\
\hline M. umbellata (L.) Hallier f. & Alves, F.M. et al. 387 (CGMS) \\
\hline \multicolumn{2}{|l|}{ Cucurbitaceae } \\
\hline Cayaponia podantha Cogn. & Salomão, A.K.D. \& Alves, F.M. 350 (CGMS) \\
\hline Momordica charantia $\mathrm{L}$. & Lescano, L.E.A.M. et al. 268 (CGMS) \\
\hline \multicolumn{2}{|l|}{ Cyperaceae } \\
\hline Cyperus aggregatus (Willd.) Endl. & Salomão, A.K.D. \& Alves, F.M. 330 (CGMS) \\
\hline C. eragrostis Lam. & Alves, F.M. et al. 391 (CGMS) \\
\hline C.s gardneri Nees & Salomão, A.K.D. \& Alves, F.M. 363 (CGMS) \\
\hline C. odoratus $\mathrm{L}$. & $\begin{array}{l}\text { Salomão, A.K.D. \& Pott, A. } 433 \text { (CGMS), Alves, F.M. et al. } 407 \\
\text { (CGMS) }\end{array}$ \\
\hline C. surinamensis Rottb. & Salomão, A.K.D. \& Pott, A. 430 (CGMS) \\
\hline C. virens Michx. & Salomão, A.K.D. \& Pott, A. 440 (CGMS) \\
\hline Eleocharis elegans (Kunth) Roem. \& Schult. & $\begin{array}{l}\text { Alves, F.M. } 435 \text { (CGMS), Salomão, A.K.D. \& Alves, F.M. } 340 \\
\text { (CGMS) }\end{array}$ \\
\hline Fimbristylis dichotoma (L.) Vahl & Salomão, A.K.D. \& Pott, A. 447 (CGMS) \\
\hline \multicolumn{2}{|l|}{ Erythroxylaceae } \\
\hline Erythroxylum cuneifolium (Mart.) O.E. Schulz & Seleme, E.P. \& Sartori, A.L.B. 109 (CGMS) \\
\hline \multicolumn{2}{|l|}{ Euphorbiaceae } \\
\hline Adelia membranifolia (Müll. Arg.) Chodat \& Hassl. & Padilha, D.R.C. et al. 15 (CGMS) \\
\hline $\begin{array}{l}\text { Cnidoscolus vitifolius var. cnicodendrum (Griseb.) Lourteig \& } \\
\text { O'Donnell * }\end{array}$ & Salomão, A.K.D. \& Alves, F.M. 379 (CGMS) \\
\hline Croton argenteus $\mathrm{L}$. & Lescano, L.E.A.M. et al. 180 (CGMS) \\
\hline C.bonplandianus Baill. & Salomão, A.K.D. \& Alves, F.M. 346 (CGMS) \\
\hline C. campestris A. St. Hill. & Alves, F.M. F. et al. 390 (CGMS) \\
\hline C. doctoris S. Moore * & Salomão, A.K.D. \& Alves, F.M. 296 (CGMS) \\
\hline C.gracilipes Baill. * & Sartori, A.L.B. \& Alves, F.M. 1082 (GMS) \\
\hline C. puberulus Müll. Arg. * & Nonato, L.M. 03 (CGMS) \\
\hline C.triqueter Lam. & Sartori, A.L.B. \& Alves, F.M. 1062 (CGMS) \\
\hline Dalechampia scandens L. & Sartori, A.L.B. \& Alves, F.M. 1061 (CGMS) \\
\hline Euphorbia hyssopifolia $\mathrm{L}$. & Marques, A.C.W. et al. 21 (CGMS) \\
\hline Jatropha aff. ribifolia (Pohl) Baill. & Alves, F.M. et al. 427 (CGMS) \\
\hline Gymnanthes discolor (Spreng.) Müll.Arg. * & Padilha, D.R.C. et al. 50 (CGMS) \\
\hline Sebastiania brasiliensis Spreng. & Seleme, E.P. et al. 305 (CGMS) \\
\hline \multicolumn{2}{|l|}{ Leguminosae } \\
\hline \multicolumn{2}{|l|}{ Caesalpinioideae } \\
\hline Bauhinia bauhinioides (Mart.) J.F. Macbr. & Seleme, E.P. \& Sartori, A.L.B. 118 (CGMS) \\
\hline B. hagenbeckii Harms & $\begin{array}{l}\text { Seleme, E.P. et al. } 281 \text { (CGMS), Sartori, A.L.B. \& Alves, F.M. } 1084 \\
\text { (CGMS) }\end{array}$ \\
\hline B. marginata D. Diet. & Alves, F.M. et al. 607 (CGMS) \\
\hline B. mollis (Bong.) D.Dietr. & Cristaldo, A.C. et al. 174 (CGMS) \\
\hline B. ungulata $\mathrm{L}$. & Nunes, G.P. et al. 71 (CGMS) \\
\hline Chamaecrista flexuosa (L.) Greene & Lescano, L.E.A.M. et al. 96 (CGMS) \\
\hline
\end{tabular}


Quadro 1. Cont.

\begin{tabular}{|c|c|}
\hline Família/Espécie & Voucher \\
\hline C. nictitans subsp. disadena (Steud.) H.S.Irwin \& Barneby & Alves, F.M. et al. 11 (CGMS) \\
\hline C.rotundifolia (Pers.) Greene & $\begin{array}{l}\text { Salomão, A.K.D. \& Alves, F.M. } 338 \text { (CGMS), Alves, F.M. et al. } 21 \\
\text { (CGMS) }\end{array}$ \\
\hline C. serpens (L.) Greene & $\begin{array}{l}\text { Salomão, A.K.D. \& Alves, F.M. } 337 \text { (CGMS), Pinto, B.E.M. } 818 \\
\text { (CGMS) }\end{array}$ \\
\hline Guibourtia hymenaefolia (Moric.) J. Léonard & Sartori, A.L.B. et al. 1019 (CGMS) \\
\hline Libidibia paraguariensis (D. Parodi) G.P. Lewis & Noguchi, D.K. 263 (CGMS) \\
\hline Parkinsonia aculeata L. & Sartori, A.L.B. et al. (CGMS 23106) \\
\hline P. praecox (Ruiz \& Pav.) J. Hawkins * & Alves, F.M. 346 (CGMS) \\
\hline Peltophorum dubium (Spreng.) Taub. & Matos-Alvos, F. 376 (CGMS) \\
\hline Poincianella marginata (Tul.) L.P.Queiroz & Noguchi, D.K. 128 (CGMS) \\
\hline P. pluviosa (DC.) L.P. Queiroz & Lescano, L.E.A.M. 221 (CGMS) \\
\hline Pterogyne nitens Tul. & Alves, F.M. et al. 57 (CGMS) \\
\hline Senna alata (L.) Roxb. & Lescano, L.E.A.M. et al. 264 (CGMS) \\
\hline S. occidentalis (L.) Link & Noguchi, D.K. et l. 217 (CGMS) \\
\hline S. obtusifolia (L.) H.S. Irwin \& Barneby & Pinto, B.E.M. 950 (CGMS) \\
\hline S. pendula (Humb. \& Bonpl. ex Willd.) H.S. Irwin \& Barneby & Noguchi, D.K. 238 (CGMS) \\
\hline S. pendula var. paludicola H.S.Irwin \& Barneby & Alves, F.M. et al. 40 (CGMS) \\
\hline S. pilifera (Vogel) H.S. Irwin \& Barneby & Salomão, A.K.D. \& Alves, F.M. 314 (CGMS) \\
\hline \multicolumn{2}{|l|}{ Mimosoideae } \\
\hline Albizia inundata (Mart.) Barneby \& J.W.Grimes & Sartori, A.L.B. 1107 (CGMS) \\
\hline A. niopoides (Spruce ex Benth.) Brenan & Noguchi, D.K. 173 (CGMS) \\
\hline Anadenanthera colubrina var. cebil (Griseb.) Altschul & $\begin{array}{l}\text { Seleme, E.P. \& Sartori, A.L.B. } 213 \text { (CGMS), Alves, F.M. \& Sartori, } \\
\text { A.L.B. } 509 \text { (CGMS) }\end{array}$ \\
\hline Chloroleucon chacoense (Burkart) Barneby \& J.W. Grimes & Alves, F.M. \& Sartori, A.L.B. 486 (CGMS) \\
\hline C. foliolosum (Benth.) G. P. Lewis * & Alves, F.M. \& Sartori, A.L.B. 536 (CGMS) \\
\hline C. tenuiflorum (Benth.) Barneby \& J.W. Grimes & Sartori, A.L.B. 1112 (CGMS) \\
\hline Desmanthus virgatus (L.) Willd. & Lescano, L.E.A.M. 176 (CGMS) \\
\hline Enterolobium contortisiliquum (Vell.) Morong & Alves, F.M. et al. 56 (CGMS) \\
\hline Microlobius foetidus var. paraguensis (Benth.) M.Sousa \& G.Andrade & Alves, F.M. 470 (CGMS), Alves, F.M. et al. 354 (CGMS) \\
\hline Mimosa bimucronata (DC.) Kuntze & Lescano, L.E.A.M. 19 (CGMS) \\
\hline M.candollei R. Grether & Nunes, G.P. et al. 297 (CGMS) \\
\hline M. centurions Barneby & Alves, F. M. et al. 25 (CGMS) \\
\hline M. debilis Willd. & Lescano, L.E.A.M. \& Nunes, G.P. 225 (CGMS) \\
\hline M. debilis var. angusta (Benth.) M. Morales \& Fortunato & Alves, F. M. et al. 28 (CGMS) \\
\hline M. debilis var. debilis Humb. \& Bonpl. ex Willd & Alves, F. M. et al. 51 (CGMS) \\
\hline M. distans Benth. var. distans & Alves, F.M. et al. 269 (CGMS) \\
\hline M. dolens Vell. & Nunes, G.P. et al. 76 (CGMS) \\
\hline M. dolens var. acerba (Benth.) Barneby & Noguchi, D.K. et al. 100 (CGMS) \\
\hline M.glutinosa Malme & Lescano, L.E.A.M. 217 (CGMS) \\
\hline M.hexandra Micheli & Seleme, E.P. et al. 335 (CGMS) \\
\hline M. pigra L. var. pigra & Lescano, L.E.A.M. et al. 277 (CGMS) \\
\hline M. polycarpa Kunth var. polycarpa & Seleme, E.P. et al. 365 (CGMS) \\
\hline M. polycarpa var. spegazzinii (Pirotta ex Hook. f.) Burkart & Nunes, G.P. et al. 184 (CGMS) \\
\hline M.sensibilis Griseb. & Alves, F.M. et al. 341 (CGMS) \\
\hline M. sensibilis var. urucumensis Barneby & Alves, F.M. et al. 274 (CGMS) \\
\hline M. somnians Humb. \& Bonpl. ex Willd. var. somnians & Lescano, L.E.A.M. et al. 19 (CGMS) \\
\hline M. xanthocentra var. subsericia (Benth.) Barneby & Nunes, G.P. et al. 45 (CGMS) \\
\hline Neptunia plena (L.) Benth. & E.S.Souza-Lima \& A.L.B. Sartori (CGMS 41378) \\
\hline N. pubescens Benth. & Salomão, A.K.D. \& Alves, F.M. 328 (CGMS) \\
\hline Parapiptadenia rigida (Benth.) Brenan & Alves, F. M. et al. 368 (CGMS) \\
\hline Piptadenia viridiflora (Kunth) Benth. * & Alves, F. M. \& Magalhães, L.C.S. 597 (CGMS) \\
\hline Prosopis alba Griseb. * & Lima, T.E. \& Sartori, A.L.B. 151 (CGMS) \\
\hline P. nigra (Griseb.)Hieron. * & Lima, T.E. \& Sartori, A.L.B. 152 (CGMS) \\
\hline P. rubriflora Hassl. * & Alves, F.M. et al. 520 (CGMS) \\
\hline P. ruscifolia Griseb. & Vargas, W. et al. 02 (CGMS) \\
\hline Samanea tubulosa (Benth.) Barneby \& J.W. Grimes & Alves, F.M. et al. 419 (CGMS) \\
\hline Senegalia lasiophylla (Benth.) Seigler \& Ebinger & F. Matos-Alves \& A.L.B. Sartori 489 \\
\hline
\end{tabular}


Quadro 1. Cont.

\begin{tabular}{|c|c|}
\hline Família/Espécie & Voucher \\
\hline Senegalia martii (Benth.) Seigler \& Ebinger * & Alves, F.M. et al. 464 (CGMS) \\
\hline S. polyphylla (DC.) Britton \& Rose & Alves, F.M. et al. 406 (CGMS) \\
\hline Vachellia caven (Molina) Seigler \& Ebinger & Noguchi, D.K. et al. 139 (CGMS) \\
\hline V. farnesiana (L.) Wight \& Arn. & Seleme, E.P. et al. 367 (CGMS) \\
\hline \multicolumn{2}{|l|}{ Papilionoideae } \\
\hline Aeschynomene brevipes Benth. & Alves, F.M. et al. 459 (CGMS) \\
\hline A. denticulata Rudd & Alves, F.M., Sartori, A.L.B., Cristaldo, A.C.M. 260 (CGMS) \\
\hline A. evenia C.Wright \& Sauvale & Sartori, A.L.B. et al. 1113 (CGMS) \\
\hline A. falcata (Poir.) DC. & $\begin{array}{l}\text { Salomão, A.K.D. \& Alves, F.M. } 377 \text { (CGMS), Alves, F.M. et al. } 270 \\
\text { (CGMS) }\end{array}$ \\
\hline A. fluminensis Vell. & Lima, L.C.P. et al. 194 (CGMS) \\
\hline A. histrix Poir. & Salomão, A.K.D. \& Alves, F.M. 329 (CGMS) \\
\hline A. mollicula Kunth & Nunes, G.P. et al. 269 (CGMS) \\
\hline A. paniculata Willd. ex Vogel & Nunes, G.P. et al. 215 (CGMS) \\
\hline A. rudis Benth. & Sartori, A.L.B. et al. 1088 (CGMS) \\
\hline Amburana cearensis (Allemão) A.C. Sm. * & Alves, F.M. et al. 342 (CGMS) \\
\hline Arachis lignosa (Chodat \& Hass1.) Krapov. \& W.C & Alves, F.M. \& Sartori, A.L.B. 504 (CGMS) \\
\hline A. microsperma Krapov., W.C. Greg. \& Valls & Pinto, B.E.M. 805 (CGMS) \\
\hline Camptosema ellipticum (Desv.) Burkart & $\begin{array}{l}\text { Sartori, A.L.B. et al. } 458 \text { (CGMS), Lescano, L.E.A.M. et al. } 14 \\
\text { (CGMS) }\end{array}$ \\
\hline Centrosema angustifolium (Kunth) Benth. & Seleme, E.P. et al. 347 (CGMS) \\
\hline C. pascuorum Mart. ex Benth. & Pinto, B.E.M. 817 (CGMS) \\
\hline Crotalaria incana $\mathrm{L}$. & Alves, F.M. et al. 30 (CGMS) \\
\hline C. incana L. var. incana & Alves, F.M. et al. 41 (CGMS) \\
\hline Desmodium incanum DC. & Noguchi, D.K. et al. 191 (CGMS) \\
\hline Discolobium pulchellum Benth. & Sartori, A.L.B. 1114 (CGMS) \\
\hline Dolichopsis paraguariensis (Benth.) Hassl. & Sartori, A.L.B. et al. 464 (CGMS) \\
\hline Galactia glaucescens Kunth & Nunes, G.P. et al. 63 (CGMS) \\
\hline G. latisiliqua Desv. * & Seleme, E.P. \& Sartori, A.L.B. 160 (CGMS) \\
\hline G. paraguariensis Chodat \& Hassl. * & Salomão, A.K.D. \& Pott, A. 411 (CGMS) \\
\hline G. striata (Jacq.) Urb. & Sartori, A.L.B. et al. 460 (CGMS) \\
\hline Geoffroea spinosa Jacq. & $\begin{array}{l}\text { Sartori, A.L.B. et al. } 463 \text { (CGMS), Alves, F.M. Sartori, A.L.B. } 472 \\
\text { (CGMS) }\end{array}$ \\
\hline Indigofera hirsuta $\mathrm{L}$. & Noguchi, D.K. et al. 23 (CGMS) \\
\hline I. microcarpa Desv. & Salomão, A.K.D. \& Alves, F.M. 393 (CGMS) \\
\hline I. spicata Forssk. & Alves, F.M. et al. 266 (CGMS) \\
\hline I. suffruticosa Mill. & Alves, F.M. et al. 410 (CGMS) \\
\hline Machaerium eriocarpum Benth. & Seleme, E.P. et al. 209 (CGMS), Sartori, A.L.B. et al. 453 (CGMS) \\
\hline Macroptilium bracteatum (Nees \& Mart.) Maréchal \& Baudet & Seleme, E.P. \& Sartori, A.L.B. 161 (CGMS) \\
\hline M. lathyroides (L.) Urb. & Nunes, G.P. et al. 265 (CGMS) \\
\hline Muellera nudiflora (Burkart) M.J.Silva \& A.M.G.Azevedo & Padilha, D.R.C. et al. 38 (CGMS), Alves, F.M. et al. 431 (CGMS) \\
\hline Rhynchosia minima (L.) DC. & Sartori, A.L.B et al. 465 (CGMS) \\
\hline Sesbania virgata (Cav.) Pers. & $\begin{array}{l}\text { Noguchi, D.K. et al. } 167 \text { (CGMS), Lescano, L.E.A.M. et al. } 185 \\
\text { (CGMS) }\end{array}$ \\
\hline Stylosanthes hamata (L.) Taub. & Nunes, G.P. et al. 266 (CGMS), Pinto, B.E.M. 733 (CGMS) \\
\hline S. maracajuensis Sousa Costa \& Van den Berg & Lescano, L.E.A.M. et al. 102 (CGMS) \\
\hline S. scabra Vogel & Noguchi, D.K. et al. 58 (CGMS) \\
\hline Tephrosia cinerea fo. pseudo-adunca Hassl. & Salomão, A.K.D. \& Sartori, A.L.B. 467 (CGMS) \\
\hline Vigna longifolia (Benth.) Verdc. & Seleme, E.P. et al. 240 (CGMS) \\
\hline V. peduncularis (Kunth) Fawc. \& Rendle & Seleme, E.P. \& Sartori, A.L.B. 169 (CGMS) \\
\hline Zornia reticulata $\mathrm{Sm}$. & Nunes, G.P. et al. 264 (CGMS), Alves, F.M. et al. 446 (CGMS) \\
\hline Hydrocharitaceae & \\
\hline $\begin{array}{l}\text { Limnobium laevigatum (Humb. \& Bonpl. ex Willd.) Heine } \\
\text { Iridaceae }\end{array}$ & Alves, F.M. et al. 58 (CGMS) \\
\hline Cipura paludosa Aubl. & Salomão, A.K.D. \& Alves, F.M. 341 (CGMS) \\
\hline Lamiaceae & \\
\hline Hyptis lappacea Benth. & Salomão, A.K.D. \& Alves, F.M. 449b (CGMS) \\
\hline Lauraceae & \\
\hline Cassytha filiformis Jacq. & Salomão, A.K.D. \& Pott, A. 398 (CGMS) \\
\hline
\end{tabular}


Quadro 1. Cont.

\begin{tabular}{|c|c|}
\hline Família/Espécie & Voucher \\
\hline \multicolumn{2}{|l|}{ Lythraceae } \\
\hline Heimia salicifolia Link & Salomão, A.K.D. \& Pott, A. 370 (CGMS \\
\hline Physocalymma scaberrimum Pohl & Padilha, D.R.C. 59 (CGMS) \\
\hline Pleurophora saccocarpa Koehne & Pott, V.J. \& Pott, A. 7825 (CGMS) \\
\hline \multicolumn{2}{|l|}{ Malpighiaceae } \\
\hline Diplopterys lutea (Griseb.) W.R. Anderson \& C. C. Davis & Ribas, C.D. et al. 10 (CGMS) \\
\hline Heteropterys dumetorum (Griseb.) Nied. * & Seleme, E.P. et al. 313 (CGMS) \\
\hline H. hypericifolia A. Juss. * & Seleme, E.P. \& Salomão, A.K.D. 396 (CGMS) \\
\hline Niedenzuella stannea (Griseb.) W.R. Anderson & Lescano, L.E.A.M. et al. 289 (CGMS) \\
\hline Thryallis laburnum S. Moore. & Francener, A. et al. 941 (CGMS) \\
\hline \multicolumn{2}{|l|}{ Malvaceae } \\
\hline Ayenia tomentosa $\mathrm{L}$. & Salomão, A.K.D. \& Alves, F.M. 292 (CGMS) \\
\hline Byttneria filipes Mart. ex K. Schum. & Salomão, A.K.D. \& Alves, F.M. 399 (CGMS) \\
\hline Ceiba samauma (Mart.) K. Schum. & Padilha, D.R.C. et al. 31 (CGMS) \\
\hline C. speciosa A. St.-Hil.) Ravenna & Padilha, D.R.C. et al. 30 (CGMS) \\
\hline Cienfuegosia drummondii (A. Gray) Lewton & Salomão, A.K.D. \& Alves, F.M. 399 (CGMS) \\
\hline Corchorus argutus Kunth & Salomão, A.K.D. \& Pott, A. 461 (CGMS) \\
\hline Guazuma ulmifolia Lam. & Nunes, G.P. et al. 35 (CGMS) \\
\hline Helicteres lhotzkyana (Schott \& Endl.) K. Schum. & Lescano, L.E.A.M. et al. 294 (CGMS) \\
\hline Malvastrum coromandelianum Garcke * & Nunes, G.P. et al. 133b (CGMS) \\
\hline Melochia graminifolia A. St.-Hil. & Salomão, A.K.D. \& Alves, F.M. 295 (CGMS) \\
\hline M. parvifolia Kunth & Nogchi, D.K. et al. 120 (CGMS) \\
\hline M. pyramidata $\mathrm{L}$. & Lescano, L.E.A.M. et al. 222b (CGMS) \\
\hline Pavonia laetevirens R.E. Fr. & Salomão, A.K.D. \& Alves, F.M. 460 (CGMS) \\
\hline P. sidifolia Kunth & Salomão, A.K.D. \& Alves, F.M. 334 (CGMS) \\
\hline Pseudobombax marginatum (A. St.-Hil., Juss. \& Cambess.) A. Robyns & Padilha, D.R.C. et al. 46 (CGMS) \\
\hline Sida cerradoensis Krap. & Salomão, A.K.D. \& Alves, F.M. 333 (CGMS) \\
\hline S. planicaulis Cav. & Nunes, G.P. et al. 273 (CGMS) \\
\hline S. linifolia Cav. & Nunes, G.P. et al. 50 (CGMS) \\
\hline Sidastrum paniculatum (L.) Fryxell & Nunes, G.P. et al. 48 (CGMS) \\
\hline Triumfetta semitriloba Jacq. & Noguchi, D.K. et al. 10 (CGMS) \\
\hline Waltheria communis A. St.-Hil. & Salomão, A.K.D. \& Pott, A. 465 (CGMS) \\
\hline Wissadula krapovickasiana Bovini & Noguchi, D.K. et al. 258 (CGMS) \\
\hline W. macrantha R.E.Fr. & Noguchi, D.K. et al. 150 (CGMS) \\
\hline W. paraguariensis Chodat & Salomão, A.K.D. \& Alves, F.M. 308 (CGMS) \\
\hline \multicolumn{2}{|l|}{ Marantaceae } \\
\hline Thalia geniculata $\mathrm{L}$. & Lescano, L.E.A.M. et al. 137 (CGMS) \\
\hline \multicolumn{2}{|l|}{ Meliaceae } \\
\hline Guarea guidonia (L.) Sleumer * & Lescano, L.E.A.M. et al. 271 (CGMS) \\
\hline Trichilia stellato-tomentosa Kuntze & Alves, F.M. \& Magalhães, L.C.S. 604 (CGMS) \\
\hline \multicolumn{2}{|l|}{ Moraceae } \\
\hline Brosimum gaudichaudii Trécul & Padilha, D.R.C. et al. 21 (CGMS) \\
\hline Maclura tinctoria (L.) D. Don ex Steud. & Voucher:Vicente, M.R. 08 (CGMS) \\
\hline \multicolumn{2}{|l|}{ Myrtaceae } \\
\hline Psidium striatulum Mart. ex DC. & Lescano, L.E.A.M. et al. 202 (CGMS) \\
\hline \multicolumn{2}{|l|}{ Nyctaginaceae } \\
\hline Boerhavia diffusa $\mathrm{L} . *$ & Noguchi, D.K. et al. 246 (CGMS) \\
\hline Neea hermaphrodita S. Moore & Seleme, E.P. \& Sartori, A.L.B. 105 (CGMS) \\
\hline Pisonia zapallo Griseb. & Pinto, A.S.C. 06 (CGMS) \\
\hline Reichenbachia paraguayensis (D. Parodi) Dugand \& Daniel & Padilha, D.R.C. et al. 47 (CGMS) \\
\hline \multicolumn{2}{|l|}{ Onagraceae } \\
\hline Ludwigia lagunae (Morong) H. Hara & Nunes, G.P. et al. 159 (CGMS) \\
\hline \multicolumn{2}{|l|}{ Orchidaceae } \\
\hline Cohniella cebolleta (Jacq.) Christenson & Salomão, A.K.D. \& Alves, F.M. 457 (CGMS) \\
\hline Sacoila lanceolata (Aubl.) Garay & Salomão, A.K.D. \& Alves, F.M. 290 (CGMS) \\
\hline \multicolumn{2}{|l|}{ Oxalidaceae } \\
\hline Oxalis cytisoides Mart. ex Zucc. & Lescano, L.E.A.M. et al. 83 (CGMS) \\
\hline O. physocalyx Zucc. ex Progel & Salomão, A.K.D. \& Alves, F.M. 360 (CGMS) \\
\hline
\end{tabular}


Quadro 1. Cont.

\begin{tabular}{|c|c|}
\hline Família/Espécie & Voucher \\
\hline \multicolumn{2}{|l|}{ Passifloraceae } \\
\hline Passiflora cincinnata Mast. & Seleme, E.P. \& Sartori, A.L.B. 219 (CGMS) \\
\hline \multicolumn{2}{|l|}{ Phyllanthaceae } \\
\hline Phyllanthus chacoensis Morong * & Seleme, E.P. \& Godoy 452 (CGMS) \\
\hline P. amarus Schumach. \& Thonn. & Salomão, A.K.D. \& Alves, F.M. 321 (CGMS) \\
\hline \multicolumn{2}{|l|}{ Phytolaccaceae } \\
\hline Microtea scabrida Urb. & Salomão, A.K.D. \& Alves, F.M. 349 (CGMS) \\
\hline Petiveria alliacea $\mathrm{L}$. & Salomão, A.K.D. \& Alves, F.M. 432 (CGMS) \\
\hline Rivina humilis $\mathrm{L}$. & Salomão, A.K.D. \& Alves, F.M. 418 (CGMS) \\
\hline \multicolumn{2}{|l|}{ Plantaginaceae } \\
\hline Monopera perennis (Hassl.) Barringer * & Nunes, G.P. et al. 245 (CGMS) \\
\hline Scoparia montevidensis (Spreng.) R.E. Fr. & Salomão, A.K.D. \& Pott, A. 446 (CGMS) \\
\hline Stemodia ericifolia K.Schum. & Noguchi, D.K. et al. 163 (CGMS) \\
\hline \multicolumn{2}{|l|}{ Poaceae } \\
\hline Aristida adscensionis L. & Salomão, A.K.D. \& Pott, A. 469 (CGMS) \\
\hline Cenchrus nervosus (Nees) Kuntze & Felismino, M.F. et al. 02 (CGMS) \\
\hline Chloris elata Desv. & Salomão, A.K.D. \& Alves, F.M. 293 (CGMS) \\
\hline C. orthonoton Döll & Felismino, M.F. et al. 01 (CGMS) \\
\hline Cynodon dactylon (L.) Pers. & Guglieri, A. 1756 (CGMS) \\
\hline Digitaria ciliaris (Retz.) Koeler & Felismino, M.F. et al. 05 (CGMS) \\
\hline D. insularis (L.) Fedde & Salomão, A.K.D. \& Pott, A. 385 (CGMS) \\
\hline Dactyloctenium aegyptium (L.) Willd. & Guglieri, A. 1758 (CGMS) \\
\hline Eragrostis bahiensis Schrad. ex Schult. & Lescano, L.E.A.M. et al. 93 (CGMS) \\
\hline E. gloeodes Ekman & Guglieri, A. 1767 (CGMS) \\
\hline Eriochloa punctata (L.) Desv. ex Ham. & Salomão, A.K.D. \& Pott, A. 436 (CGMS) \\
\hline Gouinia brasiliensis (S. Moore) Swallen & Salomão, A.K.D. 316 (CGMS) \\
\hline Gouinia paraguayensis (Kuntze) Parodi * & Salomão, A.K.D. \& Pott, A. 405 (CGMS) \\
\hline Hymenachne amplexicaulis (Rudge) Nees & Alves, F.M. et al. 374 (CGMS) \\
\hline Hyparrhenia rufa (Nees) Stapf & Guglieri, A. \& Caporal, F.J.M. 2783 (CGMS) \\
\hline Lasiacis divaricata (L.) Hitch. & Salomão, A.K.D. \& Pott, V.J. 438 (CGMS) \\
\hline Leptochloa fusca Kunth. var. uninervia (J. Presl) N.W. Snow & Salomão, A.K.D. \& Pott, A. 403 (CGMS) \\
\hline L. scabra Nees & Guglieri, A. 1765 (CGMS) \\
\hline L. virgata (L.) P. Beauv. & Salomão, A.K.D. \& Pott, A. 353 (CGMS) \\
\hline Megathyrsus maximus (Jacq.) B.K. Simon \& S.W.L. Jacobs & Salomão, A.K.D. \& Alves, F.M. 342 (CGMS) \\
\hline Melinis repens (Willd.) Zizka & Guglieri, A. \& Caporal, F.J.M. 2777 (CGMS) \\
\hline Oryza latifolia Desv. & Salomão, A.K.D. \& Pott, A. 441 (CGMS) \\
\hline Panicum dichotomiflorum Michx. & Salomão, A.K.D. \& Pott, A. 444 (CGMS) \\
\hline P. exiguum $\mathrm{Mez}$ & Guglieri, A. \& Caporal, F.J.M. 2765 (CGMS) \\
\hline P. stramineum Hitch. \& Chase & Salomão, A.K.D. \& Pott, V.J. 424 (CGMS) \\
\hline Pappophorum pappiferum (Lam.) Kuntze & Salomão, A.K.D. \& Pott, A. 331 (CGMS) \\
\hline Paspalum chacoense Parodi & Salomão, A.K.D. \& Pott, A. 319 (CGMS) \\
\hline P. plicatulum Michx. & Salomão, A.K.D. \& Pott, A. 401 (CGMS) \\
\hline P. rojasii Hack. & Guglieri, A. \& Caporal, F.J.M. 2787 (CGMS) \\
\hline P.simplex Morong & Salomão, A.K.D. \& Pott, A. 397 (CGMS) \\
\hline Rugoloa polygonata (Scrad.) Zuloaga & $\begin{array}{l}\text { Salomão, A.K.D. \& Pott, V.J. Pott } 435 \text { (CGMS), } \\
\text { Pinto, B.E.M. \& Guglieri-Caporal, A. } 224 \text { (CGMS) }\end{array}$ \\
\hline Setaria parviflora (Poir.) Kerguelén & Salomão, A.K.D. \& Alves, F.M. 320 (CGMS) \\
\hline S. nicorae Pensiero & Salomão, A.K.D. \& Alves, F.M. 396 (CGMS) \\
\hline S. setosa (Sw.) P. Beauv. & Nunes, G.P. et al. 59 (CGMS) \\
\hline S. vulpiseta (Lam.) Roem. \& Schult. & Felismino, M.F. et al. 06 (CGMS) \\
\hline Sporobolus ciliatus J. Presl. & Guglieri, A. 1762 (CGMS) \\
\hline S. indicus (L.) R. Br. var. pyramidalis (P. Beauv.) Veldkamp & Salomão, A.K.D. \& Pott, A. 434 (CGMS) \\
\hline S. monandrus Roseng., B.R. Arrill. \& Izag. & Felismino, M.F. et al. 09 (CGMS) \\
\hline S. pseudairoides Parodi & Salomão, A.K.D. \& Alves, F.M. 354 (CGMS) \\
\hline S. pyramidatus (Munro ex C. Wright) R.D. Webster & Salomão, A.K.D. \& Pott, A. 450 (CGMS) \\
\hline Steinchisma hians (Elliott) Nash & Salomão, A.K.D. 400 (CGMS) \\
\hline S. laxum (Sw.) Zuloaga & Salomão, A.K.D. \& Pott, A. 422 (CGMS) \\
\hline Tripogon spicatus (Nees) Ekman & Salomão, A.K.D. \& Pott, A 425 (CGMS) \\
\hline
\end{tabular}


Quadro 1. Cont.

\begin{tabular}{|c|c|}
\hline Família/Espécie & Voucher \\
\hline Urochloa adspersa (Trin.) R.D. Webster & Salomão, A.K.D. \& Pott, A 409 (CGMS), Guglieri, A. 1769 (CGMS) \\
\hline U. platyphylla (Munro ex C. Wright) R.D. Webster & Salomão, A.K.D. \& Pott, A. 454 (CGMS) \\
\hline \multicolumn{2}{|l|}{ Polygalaceae } \\
\hline Acanthocladus albicans A.W. Benn. K.Schum* & Padilha, D.R.C. et al. 61 (CGMS) \\
\hline Polygala molluginifolia A. St.-Hil. \& Moq. * & Salomão, A.K.D. 462 (CGMS) \\
\hline \multicolumn{2}{|l|}{ Polygonaceae } \\
\hline Coccoloba guaranitica Hassl. & Padilha, D.R.C. et al. 35 (CGMS) \\
\hline C. rigida Willd. ex Meisn. * & Seleme, E.P. \& Sartori, A.L.B. 113 (CGMS) \\
\hline C. paraguariensis Lindau * & Seleme, E.P. \& Sartori, A.L.B. 177 (CGMS) \\
\hline Polygonum punctatum Elliot & Lescano, L.E.A.M. et al. 143 (CGMS) \\
\hline Ruprechtia exploratricis Sandwith & Alves, F.M. et al. 423 (CGMS) \\
\hline R. triflora Griseb. & Seleme, E.P. \& Sartori, A.L.B. 149 (CGMS) \\
\hline Triplaris gardneriana Wedd. & Seleme, E.P. \& Sartori, A.L.B. 210 (CGMS) \\
\hline \multicolumn{2}{|l|}{ Pontederiaceae } \\
\hline Eichhornia azurea (Sw.) Kunth & Lescano, L.E.A.M. et al. 170 (CGMS) \\
\hline Pontederia cordata var. cordata L. & Lescano, L.E.A.M. et al. 188 (CGMS) \\
\hline P. cordata var. lancifolia L. * & Noguchi, D.K. et al. 166 (CGMS) \\
\hline P. subovata (Seub.) Lowden * & Nunes, G.P. et al. 271 (CGMS) \\
\hline \multicolumn{2}{|l|}{ Portulacaceae } \\
\hline Portulaca cryptantha Speg. * & Nunes, G.P. et al. 286 (CGMS) \\
\hline P. fluvialis D. Legrand & Salomão, A.K.D. \& Alves, F.M. 364 (CGMS) \\
\hline Talinum paniculatum (Jacq.) Gaertn. * & Salomão, A.K.D. \& Alves, F.M. 352 (CGMS) \\
\hline T.triangulare (Jacq.) Willd. & Salomão, A.K.D. \& Alves, F.M. 458 (CGMS) \\
\hline \multicolumn{2}{|l|}{ Rhamnaceae } \\
\hline Ziziphus mistol Griseb. * & Alves, F.M. et al. 436 (CGMS), Seleme, E.P. 307 (CGMS) \\
\hline Gouania lupuloides (L.) Urb. & Lescano, L.E.A.M. et al. 275 (CGMS) \\
\hline \multicolumn{2}{|l|}{ Rubiaceae } \\
\hline Borreria eryngioides Cham. \& Schltdl. & Salomão, A.K.D. \& Alves, F.M. 303 (CGMS) \\
\hline Calycophyllum multiflorum Griseb. & Alves, F.M. 399 (CGMS) \\
\hline Chomelia obtusa Cham. \& Schltdl. & Padilha, D.R.C. et al. 33 (CGMS) \\
\hline Genipa americana $\mathrm{L}$. & Vicente, M.R. 3 (CGMS) \\
\hline Guettarda uruguensis Cham. \& Schltdl. * & Seleme, E.P. \& Sartori, A.L.B. 173 (CGMS) \\
\hline Machaonia brasiliensis (Hoffmanns. ex Humb.) Cham. \& Schltdl. * & Padilha, D.R.C. et al. 40 (CGMS) \\
\hline Randia armata (Sw.) DC. * & $\begin{array}{l}\text { Seleme, E.P. \& Sartori, A.L.B. } 216 \text { (CGMS), Seleme, E.P. \& NEVES, } \\
\text { I.M. .378 (CGMS) }\end{array}$ \\
\hline Staelia thymoides Cham. \& Schltdl. & Salomão, A.K.D. \& Alves, F.M. 371 (CGMS) \\
\hline Tocoyena formosa (Cham. \& Schltdl.) K.Schum. * & Nunes, G.P. et al. 90 (CGMS) \\
\hline \multicolumn{2}{|l|}{ Rutaceae } \\
\hline Zanthoxylum fagara (L.) Sarg. & Seleme, E.P. et al. 318 (CGMS) \\
\hline Z. petiolare A. St.-Hil. \& Tul. & Padilha, D.R.C. et al. 57 (CGMS) \\
\hline \multicolumn{2}{|l|}{ Salicaceae } \\
\hline Casearia aculeata Jacq. & Seleme, E.P. \& Neves, I.M. 383 (CGMS) \\
\hline \multicolumn{2}{|l|}{ Santalaceae } \\
\hline Acanthosyris falcata Griseb. * & Padilha, D.R.C. et al. 14 (CGMS) \\
\hline Phoradendron affine (Pohl ex DC.) Engl. \& K. Krause & Seleme, E.P. \& Sartori, A.L.B. 227 (CGMS) \\
\hline P. liga (Gillies ex Hook. \& Arn.) Eichler & Nunes, G.P. et al. 112 (CGMS) \\
\hline \multicolumn{2}{|l|}{ Sapindaceae } \\
\hline Diplokeleba floribunda N.E. Br. & Seleme, E.P. \& Sartori, A.L.B. 215 (CGMS) \\
\hline Melicoccus lepidopetalus Radlk. & Padilha, D.R.C. et al. 43 (CGMS) \\
\hline Paullinia pinnata $\mathrm{L}$. & Seleme, E.P. \& Sartori, A.L.B. 193 (CGMS) \\
\hline Serjania erecta Radlk. & Seleme, E.P. et al. 363 (CGMS) \\
\hline S. caracasana (Jacq.) Willd. & Ribas, C.D. et al. 08 (CGMS) \\
\hline \multicolumn{2}{|l|}{ Sapotaceae } \\
\hline Sideroxylon obtusifolium (Humb. ex Roem. \& Schult.) T.D. Penn. & Padilha, D.R.C. et al. 53 (CGMS), Menegazzo, M.M. 04 (CGMS) \\
\hline \multicolumn{2}{|l|}{ Simaroubaceae } \\
\hline Castela coccinea Griseb. * & Padilha, D.R.C. et al. 29 (CGMS) \\
\hline \multicolumn{2}{|l|}{ Solanaceae } \\
\hline Grabowskia duplicata Arn. & Seleme, E.P. \& Sartori, A.L.B. 223 (CGMS) \\
\hline
\end{tabular}


Quadro 1. Cont.

\begin{tabular}{|c|c|}
\hline Família/Espécie & Voucher \\
\hline Solanum americanum Mill. & Lescanos, L.E.A.M. et al. 278 (CGMS) \\
\hline S. glaucophyllum Desf. & Salomão, A.K.D. \& Alves, F.M. 408 (CGMS) \\
\hline S. sisymbriifolium Lam. & Lescanos, L.E.A.M. et al. 236 (CGMS) \\
\hline S. viarum Dunal. & Salomão, A.K.D. \& Alves, F.M. 372 (CGMS) \\
\hline \multicolumn{2}{|l|}{ Smilacaceae } \\
\hline Smilax fluminensis Steud. & Ribas, C.D. et al. 09 (CGMS) \\
\hline \multicolumn{2}{|l|}{ Ulmaceae } \\
\hline Phyllostylon rhamnoides (J. Poiss.) Taub. & Padilha, D.R.C. 45 (CGMS) \\
\hline \multicolumn{2}{|l|}{ Verbenaceae } \\
\hline Glandularia aristigera $\mathrm{S}$. Moore & Salomão, A.K.D. \& Alves, F.M. 327 (CGMS) \\
\hline Lippia alba (Mill.) N.E.Br. & Lescano, L.E.A.M. et al. 51 (CGMS) \\
\hline L. lupulina Cham. & Lescano, L.E.A.M. et al. 33 (CGMS) \\
\hline Stachytarpheta angustifolia (Mill) Vahl. * & Salomão, A.K.D. \& Alves, F.M. 382 (CGMS) \\
\hline \multicolumn{2}{|l|}{ Violaceae } \\
\hline Pombalia calceolaria (Oken) Paula-Souza & Lescano, L.E.A.M. et al. 117 (CGMS) \\
\hline \multicolumn{2}{|l|}{ Vitaceae } \\
\hline Cissus spinosa Cambess. & Lescano, L.E.A.M. et al. 192 (CGMS) \\
\hline C. verticillata (L.) Nicolson \& C.E.Jarvis & Lescano, L.E.A.M. et al. 291 (CGMS) \\
\hline \multicolumn{2}{|l|}{ Zygophyllaceae } \\
\hline Bulnesia sarmientoi Lorentz ex Griseb. * & Alves, F.M. \& Sartori, A.L.B. 60 (CGMS) \\
\hline
\end{tabular}

\section{AGRADECIMENTOS}

Ao Conselho Nacional de Desenvolvimento Científico e Tecnológico (processo nº620176/2008-3, processo $\mathrm{n}^{\circ} 552352 / 2011-0$ ), à FUNDECT (processo $\mathrm{n}^{\circ}$ 23200.2892008) pelo suporte financeiro. Ao CNPq-INCT Herbário Virtual de Plantas e Fungos.

\section{REFERÊNCIAS}

Almeida, G. 2012. Gymnocoronis. Lista de Espécies da Flora do Brasil.

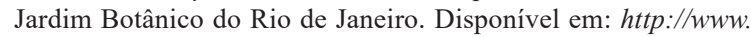
floradobrasil.jbrj.gov.br/2012/FB080668. Acessado em 07.09.2012.

Alves, F.M. 2008. Leguminosae: Caesalpinioideae e Papilionoideae de um remanescente de Chaco em Porto Murtinho-MS-Brasil. Dissertação 71 f. Universidade Federal de Mato Grosso do Sul, Mato Grosso do Sul.

Alves, F. de M. \& Sartori, A. L. B. 2009. Caesalpinioideae (Leguminosae) de um remanescente de Chaco, Porto Murtinho, MS, Brasil. Rodriguesia 60: 71-90.

Angiosperm Phylogeny Group Group III. 2009. An update of the Angiosperm Phylogeny Group classification for the orders and families of flowering plants: APG III. Botanical Journal of the Linnaean Society 161: 105-121.

Carvalho, F.S. 2011. Florística, distribuição espacial e fenologia reprodutiva de árvores e arbustos em remanescente de Chaco brasileiro. Dissertação 38 f, Universidade Federal de Mato Grosso do Sul, Mato Grosso do Sul.

Centro de Referência em Informação Ambiental -CRIA. SpeciesLink. Disponível em: http://www.splink.org.br/index. Acessado em 23.09.2012.

Hueck, K. 1972. As regiões de matas do Chaco e áreas marginais. In As florestas da América do Sul: Ecologia, Composição e Importância Econômica. Editora Polígono, Brasília, p. 240-275.

Instituto Brasileiro de Geografia e Estatística. IBGE 1992. Manual técnico da vegetação brasileira. Rio de Janeiro.

Lista de Espécies da Flora do Brasil 2012. Jardim Botânico do Rio de Janeiro. Disponível em: http://www.floradobrasil.jbrj.gov.br/2012/ FB080668. Acessado em 7.09.2012.

Lorenzi, H.1998. Árvores brasileiras. Instituto Plantarum, Nova Odessa. v. $1.384 \mathrm{p}$.
Lorenzi, H. 2009. Árvores brasileiras. Instituto Plantarum, Nova Odessa. v.3. 384 p.

Marino, G.D. \& Pensiero, J.F. 2003. Heterogeneidad florística y estructural de los bosques de Schinopsis balansae (Anacardiaceae) en el sur del Chaco húmedo. Darwiniana 41 (1-4): 17-28.

Ministério do Meio Ambiente. 2002. Avaliação e identificação de áreas e ações prioritárias para conservação, utilização e repartição de benefícios da biodiversidade brasileira. Brasília. Ministério do Meio Ambiente MMA, Brasília, 404p.

Noguchi, D.K., Nunes, G.P. \& Sartori, A.L.B. 2009. Florística e síndromes de dispersão de espécies arbóreas em remanescentes de Chaco de Porto Murtinho, Mato Grosso do Sul, Brasil. Rodriguésia 60:353-365.

Nunes, G.P. 2006. Estudo florístico de formações chaquenhas brasileiras e caracterização estrutural de um remanescente de Chaco de Porto Murtinho, MS, Brasil. Dissertação 83 f, Universidade Federal de Mato Grosso do Sul, Mato Grosso do Sul.

Padilha, D.R.C. 2011. Fitossociologia e estimativas da biomassa aérea e de carbono em Chaco Florestado no Brasil. Mestrado 71 f, Universidade Federal de Mato Grosso do Sul, Mato Grosso do Sul.

Peña-Chocarro, M.C., Juvinel, J.E., Vera, M., Maturo, H. \& Knapp, S. 2006. Guía de árboles y arbustos del Chaco Húmedo. Editora Darwin Initiative. Assunción, Paraguay. 291p.

Pott, A. \& Pott, V.J. 1994. Plantas do Pantanal. Embrapa. Brasília. 320p.

Pott, A. \& Pott, V.J. 1999. Flora do Pantanal-listagem atual das fanerógamas. In M. Dantas, J.B. Catto \& E.K. Resende (eds.). Anais do II Simpósio sobre recursos naturais sócio-econômicos do Pantanal, Embrapa Pantanal, Corumbá, p. 297-325.

Prado, D.E. 1993. What is the Gran Chaco vegetation in South America? I. A review. Contribution to the study of the flora and vegetation of the Chaco. V. Candollea. 48 (1): 145-172.

Prado, D.E., Gibbs, P.E., Pott, A. \& Pott, V.J. 1992. The Chaco-Pantanal transition in southern Mato Grosso, Brazil. Nature and Dynamics of Forest-Savanna Boundaries (P.A. Furley, J. Proctor \& J. A. Ratter, eds.), Ed. Chapman \& Hall, London, p. 451-470 .

Roic, L.D., Carrizo, E. del V., Palacio, M.O. del V. 1999. Composición de la flora de los alrededores de la ciudad de Santiago del Estero, Argentina. Quebracho 8, p. 40-46.

Salomão, A.K.D. 2009. Florística, síndromes de dispersão e similaridade de espécies de áreas chaquenhas, Mato Grosso do Sul, Brasil. Mestrado, 67 f, Universidade Federal de Mato Grosso do Sul, Mato Grosso do Sul. 
Sartori, A.L.B. 2012. The Brazilian Chaco. Glalia 4:11-18.

Seleme, E.P. 2009. Flora de remanescentes de Chaco Brasileiro: aspectos biológicos e etnobotânicos. Mestrado $48 \mathrm{f}$, Universidade Federal de Mato Grosso do Sul, Mato Grosso do Sul.

Silva, M.P., Mauro, R.A., Abdon, M. \& Silva, J.S.V. 2008. Estado de Conservação do Chaco (Savana Estépica) brasileiro. In IX Simpósio Nacional Cerrado. Brasília, DF, 6 p.

Silva, M.J. da; Tozzi, A.M.G.A. Muellera. Lista de Espécies da Flora do Brasil. Jardim Botânico do Rio de Janeiro. Disponível em: http://www.
floradobrasil.jbrj.gov.br/jabot/floradobrasi1/FB135072. Acessado em: 23.03. 2012.

Vaz, A. M.S.F. da \& Tozzi, A.M.G. de A. 2005. Sinopse de Bauhinia sect. Pauletia (Cav.) DC. (Leguminosae: Caesalpinioideae: Cercideae) no Brasil. Revista Brasileira de Botânica 28(3): 477-491.

Zak, M.R. \& Cabido, M. 2002. Spatial patterns of the Chaco vegetation of central Argentina: Integration of remote sensing and phytosociology. Applied Vegetation Science 5: 213-226. 